\title{
Entropies of Liquid Rare-Earth Metals near the Melting Point
}

\author{
H. Sasajima ${ }^{\dagger}$, T. Arai and I. Yokoyama \\ Department of Mathematics and Physics, National Defense Academy, \\ Yokosuka 239-8686, Japan
}

\begin{abstract}
In this paper, the thermodynamic entropies of the liquid rare-earth metals are explained by the statistical theory of entropy using the hypotheses about the electronic structure suggested by what is believed to be true of the corresponding solids. The pair correlation entropies are computed from measured pair correlation functions, while the triplet correlation entropies are estimated by measured structure factors with the help of the uniform fluid model for evaluation of a change in the structure factor on application of the pressure. The magnitude of the pair and triplet correlation entropies is smaller than that of the liquid $3 \mathrm{~d}$ transition metals near the melting point. The excess entropy of a hard sphere model is discussed in relation to the correlation entropies studied.
\end{abstract}

(Received November 17, 1997; In Final Form February 9, 1998)

Keywords: correlation entropy, liquid rare-earth metals, pair distribution function, structure factor

\section{Introduction}

The statistical theory for the entropy of classical liquids at equilibrium has recently been rederived by Wallace ${ }^{(1)(2)}$. The theory is based on a systematic expansion of the entropy in terms of the partial $\mathrm{N}$-particle distribution functions originally developed by $\mathrm{Green}^{(3)}$ for the canonical ensemble. Baranyai and Evans ${ }^{(4)(5)}$, however, have pointed out that Wallace's method contains some conceptual errors regarding the ensemble dependence of the entropy expansion. These authors have clarified these matters and obtained an ensemble-invariant expansion of the entropy which had been derived by Raveche $^{(6)}$ for the grand canonical ensemble. In the previous paper ${ }^{(7)}$, two of the present authors used the ensemble-invariant form derived by Baranyai and Evans, and showed that the thermodynamic entropies of the liquid $3 \mathrm{~d}$ transition metals near the melting point are well explained by this entropy expansion. In that work, the pair correlation entropies are computed from measured pair correlation functions, while the triplet correlation entropies are estimated by measured structure factors with the help of the one-component plasma model. It turned out that the triplet correlation entropy is not small enough to be completely neglected for the $3 \mathrm{~d}$ transition metals. In this paper, in order to follow the same definition as Baranyai and Evans, and also Mountain and Raveche $\tilde{e}^{(8)}$, we redefine the expressions of correlation entropies for the ion motional entropy $S_{\mathrm{I}}$ as follows:

$$
S_{\mathrm{I}}=S_{\mathrm{PG}}+S^{(2)}+S^{(3)}+\cdots
$$

where $S_{\mathrm{PG}}$ is the perfect gas entropy. We call $S^{(2)}$ the pair correlation entropy, $S^{(3)}$ the triplet correlation entropy

$\dagger$ Present address: First Aviation, First Division, Ground SelfDefense Force, Tachikawa 190-0014, Japan. and so on. The perfect gas entropy is given by

$$
S_{\mathrm{PG}}=(5 / 2)-\ln \rho \Lambda^{3} \text {. }
$$

$\rho$ is the number density of particles and $\Lambda$ is the "thermal" de Broglie wavelength. The correlation entropies are defined, respectively, by

$$
\begin{aligned}
S^{(2)}= & -\frac{1}{2} \rho \int g^{(2)}(r) \ln g^{(2)}(r) d r+\frac{1}{2} \rho \int\left(g^{(2)}(r)-1\right) d r \\
S^{(3)}= & -\frac{1}{6} \rho^{2} \iint g^{(3)}(r, s, t) \ln \delta g^{(3)}(r, s, t) d r d s \\
& +\frac{1}{6} \rho^{2} \iint\left[g^{(3)}(r, s, t)\right. \\
& -g^{(2)}(r) g^{(2)}(s)-g^{(2)}(s) g^{(2)}(t)-g^{(2)}(r) g^{(2)}(t) \\
& \left.+g^{(2)}(r)+g^{(2)}(s)+g^{(2)}(t)-1\right] d r d s
\end{aligned}
$$

where, $g^{(2)}$ and $g^{(3)}$ are the pair and triplet correlation functions and $r=|r|=\left|r_{2}-r_{1}\right|, s=|s|=\left|r_{3}-r_{1}\right|$ and $t=$ $|s-r|$. The irreducible part of the triplet correlation function is defined by

$$
\begin{aligned}
\delta g^{(3)}\left(\boldsymbol{r}_{1}, \boldsymbol{r}_{2}, \boldsymbol{r}_{3}\right)= & g^{(3)}\left(\boldsymbol{r}_{1}, \boldsymbol{r}_{2}, \boldsymbol{r}_{3}\right) /\left(g^{(2)}\left(\boldsymbol{r}_{1}, \boldsymbol{r}_{2}\right) g^{(2)}\left(\boldsymbol{r}_{1}, \boldsymbol{r}_{3}\right) g^{(2)}\right. \\
& \left.\times\left(\boldsymbol{r}_{2}, \boldsymbol{r}_{3}\right)\right) .
\end{aligned}
$$

Throughout this paper the entropy is expressed in units of $k_{\mathrm{B}}$ per particle.

The purpose of the present paper is to show the entropy calculations of the liquid rare-earth metals using the same formalism and same approximations employed in the previous paper ${ }^{(7)}$. We are particularly interested in the size of $S^{(3)}$ and also the ratio of $S^{(3)} / S^{(2)}$ in comparison with the liquid $3 \mathrm{~d}$ transition metals near the melting point. In relation to the present paper the work of Harder and Young ${ }^{(9)}$ is particularly important. These authors have explained the entropies of these metals by the hard sphere model ${ }^{(10)-(12)}$ using hypotheses about the electronic structure which closely parallel some believed 
to be correct for the corresponding solids. Both magnetic and electronic contributions appear to be crucial for correct evaluation of the total entropy. We follow their estimates for these contributions in order to stand on an equal footing with Harder and Young.

In the next section we mention an outline of the formalism and approximations to be employed. Results of numerical calculations are presented and compared with observed data in Section III. In Section IV, discussions will be given regarding the size of both $S^{(3)}$ and $S^{(3)} / S^{(2)}$, including a comment on the excess entropy. A conclusion will be given in the last section.

\section{Formalism}

Explicit expressions for the entropy are given in Ref. (7), but we outline the essential formulas for reader's convenience. In order to evaluate $S^{(3)}$, we define a function $H(r, s, t)$ as follows:

$$
H(r, s, t)=g^{(3)}(r, s, t)-g(r) g(s) g(t) .
$$

Using this new function, the first integral of $S^{(3)}$ is now approximated by

$$
\left\langle\beta \omega_{3}\right\rangle \approx-\frac{1}{6} \rho^{2} \iint H(r, s, t) d r d s .
$$

We used the notation employed by Mountain and Raveche ${ }^{(8)}$. Here we assumed that $\mid H(r, s, t) / g(r) g(s)$ $g(t) \mid \ll 1$ and $H(r, s, t)^{2} / g(r) g(s) g(t) \ll 1$ for all range of particle configurations.

Next, we consider the Fourier transform of $H(r, s, t)$ and define $\tilde{H}(Q)$ as follows:

$$
\tilde{H}(Q) \equiv \rho^{2} \int e^{i Q \cdot r} d r \int H(r, s, t) d s .
$$

Then, comparing eq. (7) with eq. (8), $\left\langle\beta \omega_{3}\right\rangle$ is given by

$$
\left\langle\beta \omega_{3}\right\rangle \approx-\frac{1}{6} \rho^{2} \int d r \int H(r, s, t) d s=-\frac{1}{6} \tilde{H}(0) .
$$

For convenience of discussion, $\tilde{H}(Q)$ is separated into three parts:

$$
\tilde{H}(Q)=H_{1}(Q)+H_{2}(Q)+H_{3}(Q),
$$

where

$$
\begin{aligned}
& H_{1}(Q)=\frac{1}{(2 \pi)^{3} \rho}[S(Q)-1] *[S(Q)-1]^{2}, \\
& H_{2}(Q)=[S(Q)-1][S(0)+S(Q)-1],
\end{aligned}
$$

and

$$
H_{3}(Q)=-\rho k_{\mathrm{B}} T\left(\frac{\partial S(Q)}{\partial p}\right)_{T} .
$$

In the above equations $S(Q)$ is the structure factor, and * means the convolution between $[S(Q)-1]$ and $[S(Q)$ $-1]^{2}$. A change in the structure factor on application of the pressure $p$ is required for $H_{3}(Q)$ but this quantity is generally not available in the literature. In order to estimate the $Q=0$ value of the function $H_{3}(Q)$ we have em- ployed the one-component plasma model ${ }^{(13)}$ in Ref. (7). It turned out that the value of the entropy arising from $H_{3}(0)$ is negligibly small (nearly zero) in the present entropy analysis. The same conclusion can be obtained even if we use the uniform fluid model proposed by Egelstaff and coworkers ${ }^{(14)(15)}$. According to these authors, if, as in a crystal, a hydrostatic compression of the liquid merely produces a uniform decrease in the interatomic distances, without any change in the average relative positions of the atoms, the pair correlation function will depend on $r \rho^{1 / 3}$, and not on $r$ and $\rho$ separately, so that

$$
H_{3}(Q) \doteqdot(S(0) / 3) Q\left(\frac{\partial S(Q)}{\partial Q}\right)_{T, \rho} .
$$

Because of the presence of $Q$ in the right hand side of eq. (14), $H_{3}(Q=0)=0$, and it turns out that $\left\langle\beta \omega_{3}\right\rangle=$ $-(1 / 6) \tilde{H}(0)=-(1 / 6)\left(H_{1}(0)+H_{2}(0)\right)$ from this model too. For further details of the volume-dependence of the structure factors please see Ref. (16). The second integral of $S^{(3)}$ is readily evaluated by using eq. (30) of Ref. (7).

\section{Results}

For liquid $\mathrm{La}, \mathrm{Ce}, \mathrm{Pr}, \mathrm{Nd}, \mathrm{Eu}, \mathrm{Gd}, \mathrm{Tb}$ and $\mathrm{Yb}$ near the melting point, Fig. 1 shows the pair correlation functions $g(r)$ and the corresponding pair correlation entropy $S^{(2)}$ $(r)$ as a function of $r . S^{(2)}(r)$ was not estimated as the sum of two integrals of eq. (3). Although the second integral of eq. (3) is easily estimated by eq. (29) of Ref. (7), the value of the first integral of eq. (3) is difficult to calculate exactly, yet predominant in evaluating the total entropy. In order to work out $S^{(2)}$ accurately, we calculated $S^{(2)}$ by performing one integration so as to cancel errors due to oscillations of the two integrands ${ }^{(4)(5)}$. However, we estimated errors arising from this source. The errors are shown in Table 1. As is seen from Fig. $1, S^{(2)}(r)$ is thought to be almost constant at about $1.5 \mathrm{~nm}$ in $r$ or so. In the present work all calculations were performed using the experimental data due to Waseda ${ }^{(18)}$, and Waseda and Ueno ${ }^{(19)}$. The three-body terms will not need to be evaluated with the same relative accuracy as that required for the two-body terms. The convolution terms $H_{1}(Q)$ are shown in Fig. 2. It is recognized from the $H_{1}(Q)$ function that a broad negative minimum is seen around $Q \approx 0.4$ a.u. ${ }^{-1}$ for lighter rare earths, but this position of minimum seems to shift towards the high- $Q$ side with increasing number of 4 f-electrons ( $Q \approx 0.6$ a.u. ${ }^{-1}$ for liquid $\mathrm{Yb}$ ). What we are interested in in the present work is the $Q=0$ value of the $H_{1}(Q)$. As is seen from the figure, the value of $H_{1}(0)$ appears to be getting bigger, clearly except $\mathrm{Ce}$ and $\mathrm{Gd}$, with increasing number of $4 \mathrm{f}$-electrons. Incidentally, all $H_{1}(0)$ values were obtained by using the exact limiting formula

$$
H_{1}(0)=\frac{2}{(2 \pi)^{2} \rho} \int_{0}^{\infty}[S(Q)-1]^{3} Q^{2} d Q .
$$

Before going to show numerical details of the entropy calculations, we must mention about the electronic and 

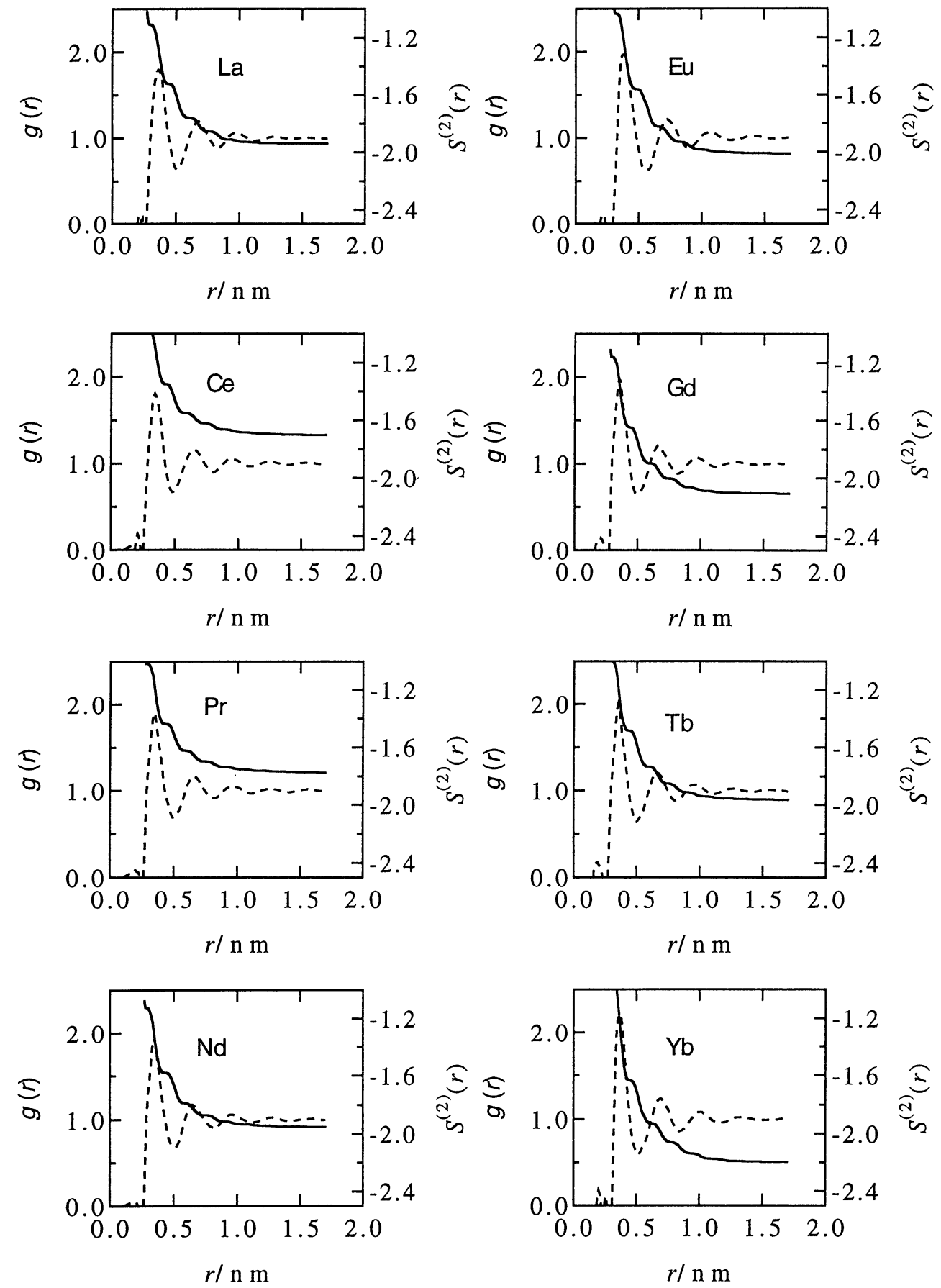

Fig. 1 The pair correlation functions, $g(r)$, and the pair correlation entropies, $S^{(2)}(r)$ (in units of $N k_{\mathrm{B}}$ ), for eight rare-earth metals near the melting point as a function of $r$. Solid line is for $S^{(2)}(r)$ (right scale) and broken line for $g(r)$ (left scale).

Table 1 The entropies of the liquid rare-earth metals (in units of $N k_{\mathrm{B}}$ ) arising from the various sources. The values of the excess entropy, $S_{\text {pack }}$, are taken from Ref. (9). Experimental data, $S_{\text {expt }}$, are due to Hultgren et al. ${ }^{(17)}$.

\begin{tabular}{|c|c|c|c|c|c|c|c|c|c|c|c|}
\hline & $T / \mathrm{K}$ & $S_{\mathrm{PG}}$ & $S^{(2)}$ & $S^{(3)}$ & $S_{\text {elec }}$ & $S_{\text {mag }}$ & $S_{\text {calc }}$ & $S_{\text {expt }}$ & $\begin{array}{c}S^{(3)} / S^{(2)} \\
(\%)\end{array}$ & $S^{(2)}+S^{(3)}$ & $S_{\text {pack }}$ \\
\hline $\mathrm{La}$ & 1243 & 15.7 & $-1.9 \pm 0.1$ & -0.4 & 0.5 & 0 & 13.9 & 13.0 & 21 & -2.3 & -3.6 \\
\hline $\mathrm{Ce}$ & 1143 & 15.5 & $-1.7 \pm 0.2$ & -0.2 & 0.6 & 1.8 & 16.0 & 14.4 & 12 & -1.9 & -3.4 \\
\hline $\mathrm{Pr}$ & 1223 & 15.6 & $-1.9 \pm 0.2$ & -0.3 & 0.4 & 2.2 & 16.0 & 15.8 & 16 & -2.2 & -3.6 \\
\hline $\mathrm{Nd}$ & 1323 & 15.7 & $-1.9 \pm 0.2$ & -0.5 & 0.5 & 2.3 & 16.1 & 15.9 & 26 & -2.4 & -3.6 \\
\hline $\mathrm{Eu}$ & 1103 & 16.0 & $-2.0 \pm 0.2$ & -0.6 & 0.3 & 2.1 & 15.8 & 15.6 & 30 & -2.6 & -3.4 \\
\hline $\mathrm{Gd}$ & 1603 & 16.2 & $-2.1 \pm 0.1$ & -0.5 & 1.0 & 2.1 & 16.7 & 15.6 & 24 & -2.6 & -3.6 \\
\hline $\mathrm{Tb}$ & 1653 & 16.2 & $-2.0 \pm 0.2$ & -0.6 & 1.1 & 2.6 & 17.3 & 16.8 & 30 & -2.6 & -3.6 \\
\hline $\mathrm{Yb}$ & 1123 & 16.0 & $-2.2 \pm 0.2$ & -0.8 & 0.3 & 0 & 13.3 & 13.0 & 36 & -3.0 & -3.6 \\
\hline
\end{tabular}



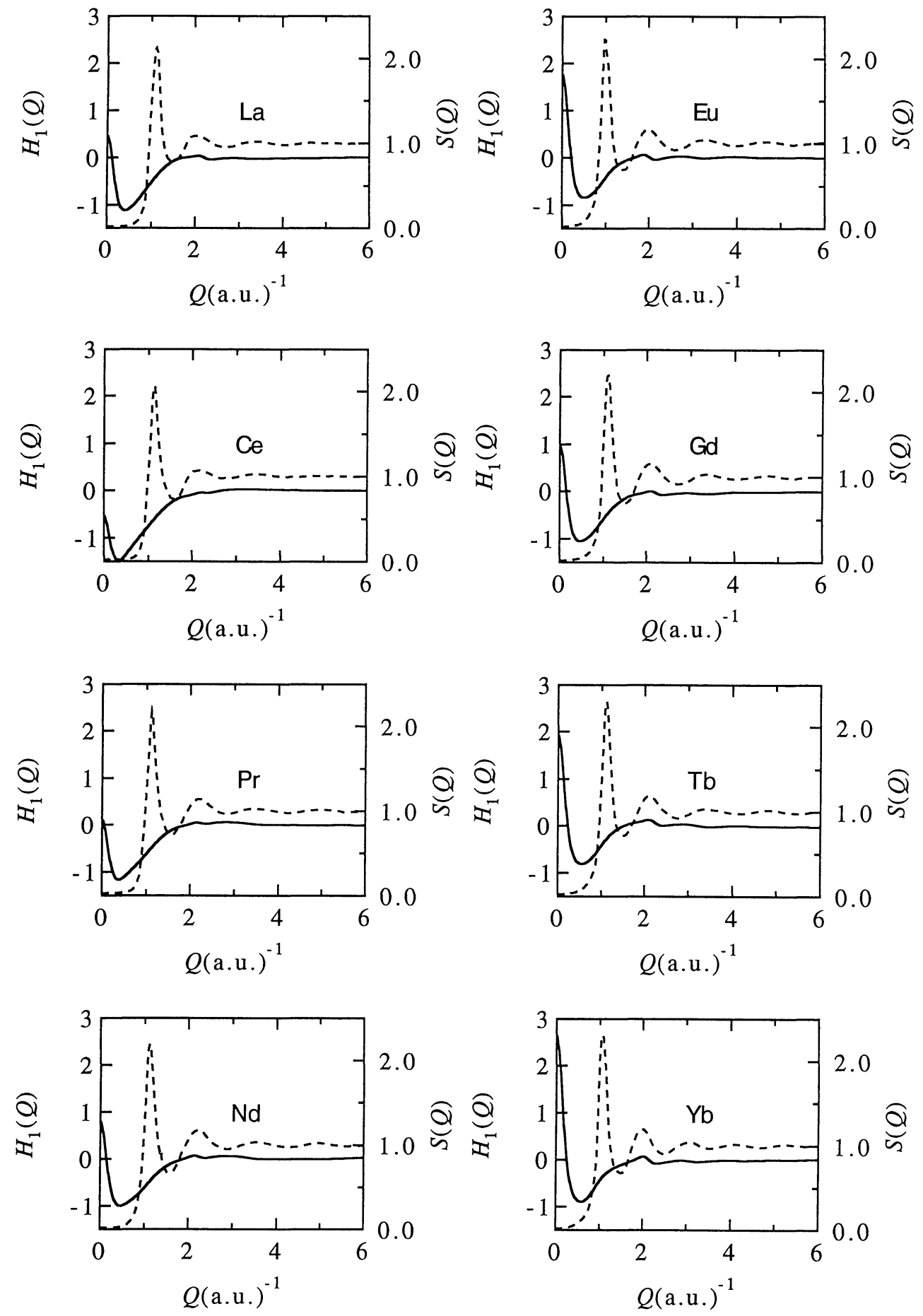

Fig. 2 The convolution terms, $H_{1}(Q)$, for the metals shown in Fig. $1\left(1\right.$ a.u. $\left.{ }^{-1} \approx 18.9 \mathrm{~nm}^{-1}\right)$. Solid line is for $H_{1}(Q)$ (left scale) and broken line for $S(Q)$ (right scale).

the magnetic contributions to the total entropy. The total entropy in the present formalism is given by

$$
S=S_{\mathrm{I}}+S_{\text {elec }}+S_{\text {mag }}
$$

where $S_{\mathrm{I}}$ is given by eq. (1), $S_{\text {elec }}$ denotes the electronic entropy expressed by

$$
S_{\text {elec }}=(1 / 3) \pi^{2} N\left(E_{\mathrm{F}}\right) k_{\mathrm{B}} T .
$$

Here $N\left(E_{\mathrm{F}}\right)$ is the density of states at the Fermi energy and should be for the liquid state but no information, theoretical or experimental, exists on this quantity, we use the data for the corresponding solids which is listed in the table of Harder and Young ${ }^{(9)}$. The values of $S_{\text {elec }}$ are recalculated and given in Table 2 . Next, $S_{\text {mag }}$ denotes the magnetic entropy expressed by

$$
S_{\mathrm{mag}}=\ln (2 J+1) .
$$

Here $J$ stands for the quantum number of the total angular momentum of the ground states for rare earth ions. At the temperatures of interest, it is natural to assume 
Table 2 The electronic entropy, $S_{\text {elec }}$, in units of $N k_{\mathrm{B}}$. All metals are allocated three valence electrons per atom except Eu and $\mathrm{Yb}$. The values of the $N\left(E_{\mathrm{F}}\right)$ are taken from Ref. (9).

\begin{tabular}{cccc}
\hline \hline Ion & Ground state & $\begin{array}{c}N\left(E_{\mathrm{F}}\right) \\
\text { (states/atom ry) }\end{array}$ & $S_{\text {elec }}$ \\
\hline $\mathrm{La}^{3+}$ & ${ }^{1} \mathrm{~S}_{0}$ & 19.4 & 0.5 \\
$\mathrm{Ce}^{3+}$ & ${ }^{2} \mathrm{~F}_{5 / 2}$ & 23.5 & 0.6 \\
$\mathrm{Pr}^{3+}$ & ${ }^{3} \mathrm{H}_{4}$ & 16.2 & 0.4 \\
$\mathrm{Nd}^{3+}$ & ${ }^{4} \mathrm{I}_{9 / 2}$ & 16.8 & 0.5 \\
$\mathrm{Eu}^{2+}$ & ${ }^{8} \mathrm{~S}_{7 / 2}$ & 12.0 & 0.3 \\
$\mathrm{Gd}^{3+}$ & ${ }^{8} \mathrm{~S}_{7 / 2}$ & 28.5 & 1.0 \\
$\mathrm{~Tb}^{3+}$ & ${ }^{7} \mathrm{~F}_{6}$ & 32.4 & 1.1 \\
$\mathrm{Yb}^{2+}$ & ${ }^{1} \mathrm{~S}_{0}$ & 1.1 & 0.3 \\
\hline \hline
\end{tabular}

Table 3 The magnetic entropy, $S_{\text {mag }}$, in units of $N k_{\mathrm{B}} . S$ denotes the total spin angular-momentum quantum number, $L$ the total orbital angular-momentum quantum number and $J$ the total angularmomentum quantum number, respectively.

\begin{tabular}{ccccl}
\hline \hline Ion & $S$ & $L$ & $J$ & $S_{\text {mag }}$ \\
\hline $\mathrm{La}^{3+}$ & 0 & 0 & 0 & 0 \\
$\mathrm{Ce}^{3+}$ & $1 / 2$ & 3 & $5 / 2$ & 1.8 \\
$\mathrm{Pr}^{3+}$ & 1 & 5 & 4 & 2.2 \\
$\mathrm{Nd}^{3+}$ & $3 / 2$ & 6 & $9 / 2$ & 2.3 \\
$\mathrm{Eu}^{2+}$ & $7 / 2$ & 0 & $7 / 2$ & 2.1 \\
$\mathrm{Gd}^{3+}$ & $7 / 2$ & 0 & $7 / 2$ & 2.1 \\
$\mathrm{~Tb}^{3+}$ & 3 & 3 & 6 & 2.6 \\
$\mathrm{Yb}^{2+}$ & 0 & 0 & 0 & 0 \\
\hline \hline
\end{tabular}

that the $2 J+1$ levels of the ground state multiplet are essentially equally populated by that the occupancies of excited multiplets are effectively zero. Then we may write $S_{\text {mag }}$ as eq. (18) in units of $N k_{\mathrm{B}}$. The values of $S_{\text {mag }}$ so found are given in Table 3. Finally, numerical details of the entropy calculated in this work are summarized in Table 1 together with the observed values ${ }^{(17)}$ for the purpose of comparison.

\section{Discussion}

First of all, on comparing the present Table 1 with Table 1 of Ref. (7), the absolute values of both $S^{(2)}$ and $S^{(3)}$ are generally smaller than those of the liquid 3d transition metals (Please note that in Table 1 of Ref. (7) $S^{(2)}$ + eq. (29) and $S^{(3)}+$ eq. (30) equal, respectively, $S^{(2)}$ and $S^{(3)}$ in the present paper). However, the ratio of $S^{(3)} / S^{(2)}$ is around 0.25 (obviously except $\mathrm{Ce}$ ). For the liquid $3 \mathrm{~d}$ transition metals except $\mathrm{Mn}$ this ratio was from 0.25 to 0.30 . For heavier rare earths, as is seen from the column 10 , the ratio of $S^{(3)} / S^{(2)}$ becomes much the same value as that of the $3 \mathrm{~d}$ metals with increasing number of $4 \mathrm{f}$-electrons. Regarding the absolute values of $S^{(2)}$ and $S^{(3)}$ the difference between the rare-earths and $3 \mathrm{~d}$ metals arises mainly from the difference in the first-peak-height region of the experimental structural data. When we look at the $g(r)$ and $S(Q)$ data of Waseda the first-peak heights of both $g(r)$ and $S(Q)$ for the rare-earth metals are generally lower than those of the $3 \mathrm{~d}$ metals. For $S^{(2)}$ small values of $g(r)$ directly lead to the small absolute values of $S^{(2)}$ from eq. (3). For $S^{(3)}$ small values of $S(Q)$ are reflected to the size of $S^{(3)}$ through eq. (15) since the first integral of eq. (4) is approximated by $\left\langle\beta \omega_{3}\right\rangle=-(1 / 6) \tilde{H}(0)=$ $-(1 / 6)\left(H_{1}(0)+H_{2}(0)\right)$. In any case, $S^{(3)}$ is not small enough to be neglected as was pointed out in the $3 \mathrm{~d}$ metals. Secondly, an interesting fact emerges from the present entropy calculations. The value of column 11 corresponds to the total excess entropy and this should be compared with the $S_{\text {pack }}$ which was taken from Harder and Young ${ }^{(9)}$. As is seen from the comparison, the absolute values of the calculated total excess entropies are always smaller than those of the hard-sphere model. In relation to this point we recall the paper of Young ${ }^{(10)}$ who proposed an empirical equation of the entropy:

$$
S=S_{\mathrm{HS}}+S_{\mathrm{S}},
$$

where $S_{\mathrm{S}}$ is an entropy contribution arising from the softness of interatomic forces, and $S_{\mathrm{Hs}}$ is, now generalizing to include the magnetic term, given as

$$
S_{\mathrm{HS}}=S_{\mathrm{PG}}+S_{\text {pack }}+S_{\text {elec }}+S_{\mathrm{mag}} \text {. }
$$

Here $S_{\text {pack }}$ is the excess entropy, best described via the Carnahan-Starling expression ${ }^{(20)}$. Young concluded in Ref. (10) that $S_{\mathrm{S}}$ is always positive. In the present entropy analysis $S^{(2)}+S^{(3)}$ correspond to $S_{\text {pack }}+S_{\mathrm{S}}$, so that in the light of both the relative softness of the rare earth cores ${ }^{(21)}$ and the calculated values given in column 11 Young's conclusion appears to be well supported by the present entropy study. Finally, agreement between the calculated total entropies and the experimental data is rather good on considering that our evaluation of the total entropy contains some hypotheses, approximations and relies solely on the observed structural data which might contain some errors. When we look more closely at the $S_{\text {calc }}$ and the $S_{\text {expt }}$, however, it is recognized that $S_{\text {calc }} \geq S_{\text {expt }}$. The fact that $S_{\text {calc }} \geq S_{\text {expt }}$ suggests to us that an entropy contribution of a quarter of $S^{(3)}$ or so might stem from the higher order expansion terms for $n \geq 4$. This is because the higher order correlations are to reduce the freedom of the ion motion, hence to decrease the entropy. As a result, a better agreement between theory and experiment would be expected by inclusion of this higher-order effect, though it is difficult for each metal to estimate this effect quantitatively.

\section{Conclusion}

The conclusion drawn from the present study is as follows:

(1) The thermodynamic entropies of the liquid rareearth metals are explainable by the statistical theory of entropy expressed by eq. (1) using assumptions about the electronic structure suggested by what is believed to be true of the corresponding solids.

(2) The absolute values of both $S^{(2)}$ and $S^{(3)}$ are generally smaller than those of the liquid $3 \mathrm{~d}$ transition metals. For heavier rare earths, however, the ratio of $S^{(3)} / S^{(2)}$ becomes much the same value as that of the $3 \mathrm{~d}$ metals with increasing number of $4 \mathrm{f}$-electrons. 
(3) The present work as well as the previous work ${ }^{(7)}$ suggest that the expansion of the entropy of eq. (1) converges less rapidly than might have been expected from an analysis of the two-body term alone $e^{(1)(2)}$.

\section{Acknowledgment}

One of the authors (IY) is grateful to Mr. Hiroki Yokoyama for his assistance in the calculation of RusellSaunders coupling.

\section{REFERENCES}

(1) D. C. Wallace: Phys. Letters A, 122 (1987), 418.

(2) D. C. Wallace: Proc. R. Soc. Lond. A, 433 (1991), 615.

(3) H. S. Green: The Molecular Theory of Fluids, North-Holland, Amsterdam, (1952), p. 70.

(4) A. Baranyai and D. J. Evans: Phys. Rev. A, 40 (1989), 3817

(5) A. Baranyai and D. J. Evans: Phys. Rev. A, 42 (1990), 849.

(6) H. J. Raveché: J. Chem. Phys., 55 (1971), 2242.

(7) I. Yokoyama and T. Arai: Mater. Trans., JIM, 37 (1996), 527.

(8) R. D. Mountain and H. J. Raveché: J. Chem. Phys., 55 (1971), 2250.

(9) J. M. Harder and W. H. Young: Phys. Letters, 61A (1977), 468.
(10) W. H. Young: Liquid Metals 1976, ed. by R. Evans and D. A. Greenwood, Institute of Physics Conference Series No. 30, (1977), p. 1.

(11) M. Shimoji: Liquid Metals, Academic Press, London, (1977), p. 15.

(12) N. W. Ashcroft and D. Stroud: Solid State Physics, Academic Press, New York, 33 (1978), p. 1.

(13) M. Baus and J. P. Hansen: Phys. Rep., 59 (1980), 1.

(14) P. A. Egelstaff, D. I. Page and C. R. T. Heard: J. Phys. C, 4 (1971), 1453.

(15) P. T. Cummings and P. A. Egelstaff: J. Phys. F, 12 (1982), 233.

(16) I. Yokoyama and W. H. Young: Phys. Chem. Liq., 14 (1985), 163.

(17) R. Hultgren, P. D. Desai, D. T. Hawkins, M. Gleiser, K. K. Kelly and D. Wagman: Selected Values of the Thermodynamic Properties of the Elements, Metals Park, Ohio, ASM, (1973), p. 118 , p. 172 , p. 201 , p. 278 , p. 344, p. 393, p. 499 and p. 563.

(18) Y. Waseda: The Structure of Non-Crystalline Materials, McGrawHill, New York, (1980), p.254, p. 278 and p. 282.

(19) Y. Waseda and S. Ueno: Sci. Rep. Res. Inst. Tohoku University, 34A (1987), 15.

(20) N. F. Carnahan and K. E. Starling: J. Chem. Phys., 51 (1969), 635.

(21) I. Yokoyama, S. Naito and Y. Waseda: J. Less-Common Metals, 136 (1987), 25 